\title{
A tunable millimeter-wave phase shifter driven by dielectric elastomer actuators
}

\author{
O. A. Araromi, P. Romano, S. Rosset, J. Perruisseau-Carrier, H. R. Shea
}

Proc. SPIE 9056, Electroactive Polymer Actuators and Devices (EAPAD) 2014, 90562M (March 13, 2014); doi: $10.1117 / 12.2044589$

Copyright 2013 Society of Photo-Optical Instrumentation Engineers. One print or electronic copy may be made for personal use only. Systematic electronic or print reproduction and distribution, duplication of any material in this paper for a fee or for commercial purposes, or modification of the content of the paper are prohibited.

http://dx.doi.org/10.1117/12.2044589 


\title{
A tunable millimeter-wave phase shifter driven by dielectric elastomer actuators
}

\author{
O. A. Araromi*a ${ }^{\text {a }}$ P. Romano ${ }^{\mathrm{b}}$, S. Rosset ${ }^{\mathrm{a}}$, J. Perruisseau-Carrier ${ }^{\mathrm{b}}$, H. R. Shea ${ }^{\mathrm{a}}$ \\ ${ }^{a}$ Microsystems For Space Technologies Laboratory, École Polytechnique Fédérale de Lausanne,

 \\ Lausanne, Lausanne, Switzerland
}

\begin{abstract}
We present the successful operation of the first dielectric elastomer actuator (DEA) driven tunable millimeter-wave phase shifter. The development of dynamically reconfigurable microwave/millimeter-wave (MW/MMW) antenna devices is becoming a prime need in the field of telecommunications and sensing. The real time updating of antenna characteristics such as coverage or operation frequency is particularly desired. However, in many circumstances currently available technologies suffer from high EM losses, increased complexity and cost. Conversely, reconfigurable devices based on DEAs offer low complexity, low electromagnetic (EM) losses and analogue operation. Our tunable phase shifter consists of metallic strips suspended a fixed distance above a coplanar waveguide (CPW) by planar DEAs. The planar actuators displace the metallic strips $(10 \mathrm{~mm}$ in length) in-plane by $500 \mu \mathrm{m}$, modifying the EM field distribution, resulting in the desired phase shift. The demanding spacing (50 $\pm 5 \mu \mathrm{m}$ between CPW and metallic strips) and parallel alignment criteria required for optimal device operation are successfully met in our device design and validated using bespoke methods. Our current device, approximately $60 \mathrm{~mm} \times 60 \mathrm{~mm}$ in planar dimensions, meets the displacement requirements and we observe a considerable phase shift $\left(\sim 95^{\circ}\right.$ at $\left.25 \mathrm{GHz}\right)$ closely matching numerical simulations. Moreover, our device achieves state of the art performance in terms of phase shift per EM loss $\sim 235^{\circ} / \mathrm{dB}$ $(35 \mathrm{GHz})$, significantly out performing other phase shifter technologies, such as MMIC phase shifters.
\end{abstract}

Keywords: Planar dielectric elastomer actuators (DEAs), tunable MMW phase shifter, reconfigurable MMW devices

\section{INTRODUCTION}

Wireless communication systems (e.g. Wifi networks, cell phones, satellite communications) integrate antenna devices necessary to control electromagnetic (EM) wave transmission and/or reception. Reconfigurable antenna devices operating in the Microwave (MW) and Millimeter-wave (MMW) range, typically used in satellite communications, are increasing in need due to their ability to dynamically update their characteristics, such as coverage, polarization or operating frequency ${ }^{1}$. Reconfigurable phase shifters are key components in MW and MMW phased array antennas and radar systems, and are currently realised with several technologies including monolithic microwave integrated circuit (MMIC), ferromagnetic, and RF-MEMS ${ }^{2}$. However, in many circumstances these technologies suffer from high EM losses, increased complexity, and cost, which are all key selection factors with this technology ${ }^{3}$. For example, reconfigurable MMIC devices are reliable with speedy operation but typically suffer large EM losses ( $>5 \mathrm{db})$ at $\mathrm{MMW}^{4,5}$.

Here, we present a novel approach which realizes reconfigurable phase shifting by mechanically displacing EM field modifying elements using planar dielectric elastomer actuators (DEAs). DEAs consist of an elastomer membrane sandwiched between two compliant electrodes, the subsequent application of an electric field $(\sim 70 \mathrm{~V} / \mu \mathrm{m})$ across the electrodes results in thickness compression and large (over $200 \%$ in area ${ }^{6}$ ) in-plane expansion. Moreover, once the voltage is applied no additional power is required to maintain actuation excluding that related to the very small leakage current through the elastomer membrane. These features make DEAs an ideal candidate for phase shifter devices, as also does the low cost of fabricating devices, low device complexity, reduced EM signal losses, reduced size and bulkiness, low power consumption and analogue operation. Our device design utilizes linear in-plane expansion of DEAs to displace loading elements within the EM active region of our device, thus inducing true time delay phase shifting. To our knowledge, this is first reconfigurable phase shifter based on DEA technology and paves the way for a host of other reconfigurable MMW devices based on DEAs.

Electroactive Polymer Actuators and Devices (EAPAD) 2014, edited by Yoseph Bar-Cohen, Proc. of SPIE Vol. 9056, 90562M - (C) 2014 SPIE · CCC code: 0277-786X/14/\$18 - doi: 10.1117/12.2044589 


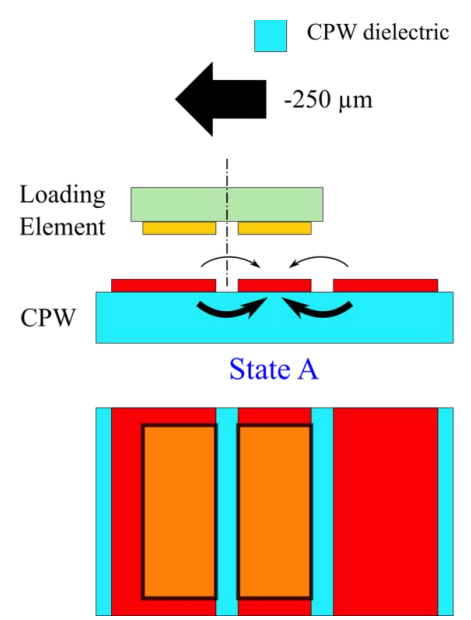

(a)

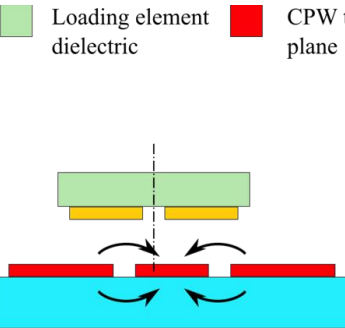

Central State



(b)
CPW transmission $\square$ Metallic strips

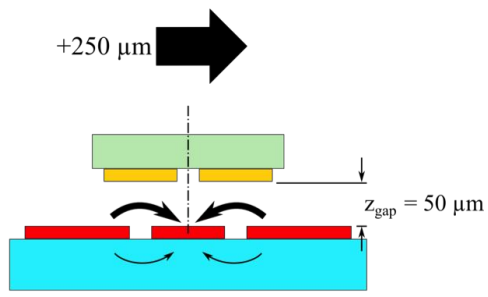

State B



(c)

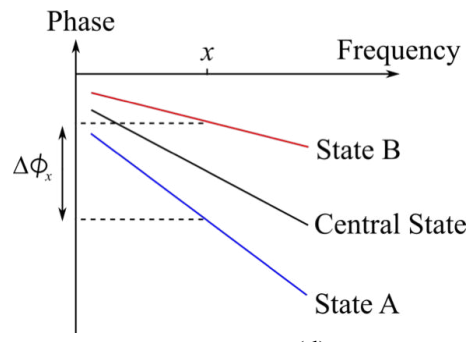

(d)

Figure 1. Schematic representation of the phase shifter working principle. A coplanar waveguide (bottom) is loaded with metallic (copper) lines, which modify the electric field distribution in the CPW dielectric and the air gap. The horizontal position of the loading lines relative to the CPW determine how the field is distributed. (a) and (c) represent the position of the device at the extremes of displacement relative to the Central State (b) with the EM field more concentrated in the dielectric substrate and the air gap, respectively (Top figures indicate side view, bottom figures indicate plan view with the loading element dielectric removed for clarity). The change in field distribution results in a change in the propagation constant $\beta$, which in turn produces a phase shift $\Delta \phi$ by Equation (1). A vertical spacing $z_{\text {gap }}=50 \mu \mathrm{m}$ is required for our design. (d) A schematic representation of the phase vs. frequency relationship for the State A, State B and the Central State (the maximum phase change $\Delta \phi_{x}$ at a given frequency $x$ indicated).

\section{DEVICE DESIGN}

The phase shifter operating principle can be seen in Figure 1. A $50 \Omega$ coplanar waveguide (CPW) carrying the EM signal is loaded by suspending thin strips of metal (loading lines) a fix distance above its surface. A change in the lateral position of the loading lines relative to the Central State (Figure 1(b)) modifies the electric field distribution: a left movement increases the field within the high permittivity dielectric (Figure 1(a)), while a right movement increases the electric field distribution in the air gap between the metallic loading lines and the CPW (Figure 1(c)). This change in electric field concentration produces a change in the propagation constant $\beta$ of the transmission line (TL), which in turn generates a change in phase, given by ${ }^{7}$ :

$$
\Delta \phi(A \rightarrow B)=-\left(\beta_{B}-\beta_{A}\right) L_{\phi} .
$$

where $L_{\phi}$ is the length of the of the movable loading elements and the subscripts A and B represent the extremes State A and State B (Figure 1). This EM design has many appealing advantages including: 1) the maximum phase change produced is linearly proportional to the length of the metallic loading lines $L_{\phi}$ (true-time-delay principle), hence the required phase change can be easily selected by the designer. 2) The actuation part is mechanically decoupled from the $\mathrm{TL}$, hence comparatively low loss operation is possible relative to other reconfiguration technologies ${ }^{7}$. The EM concept 


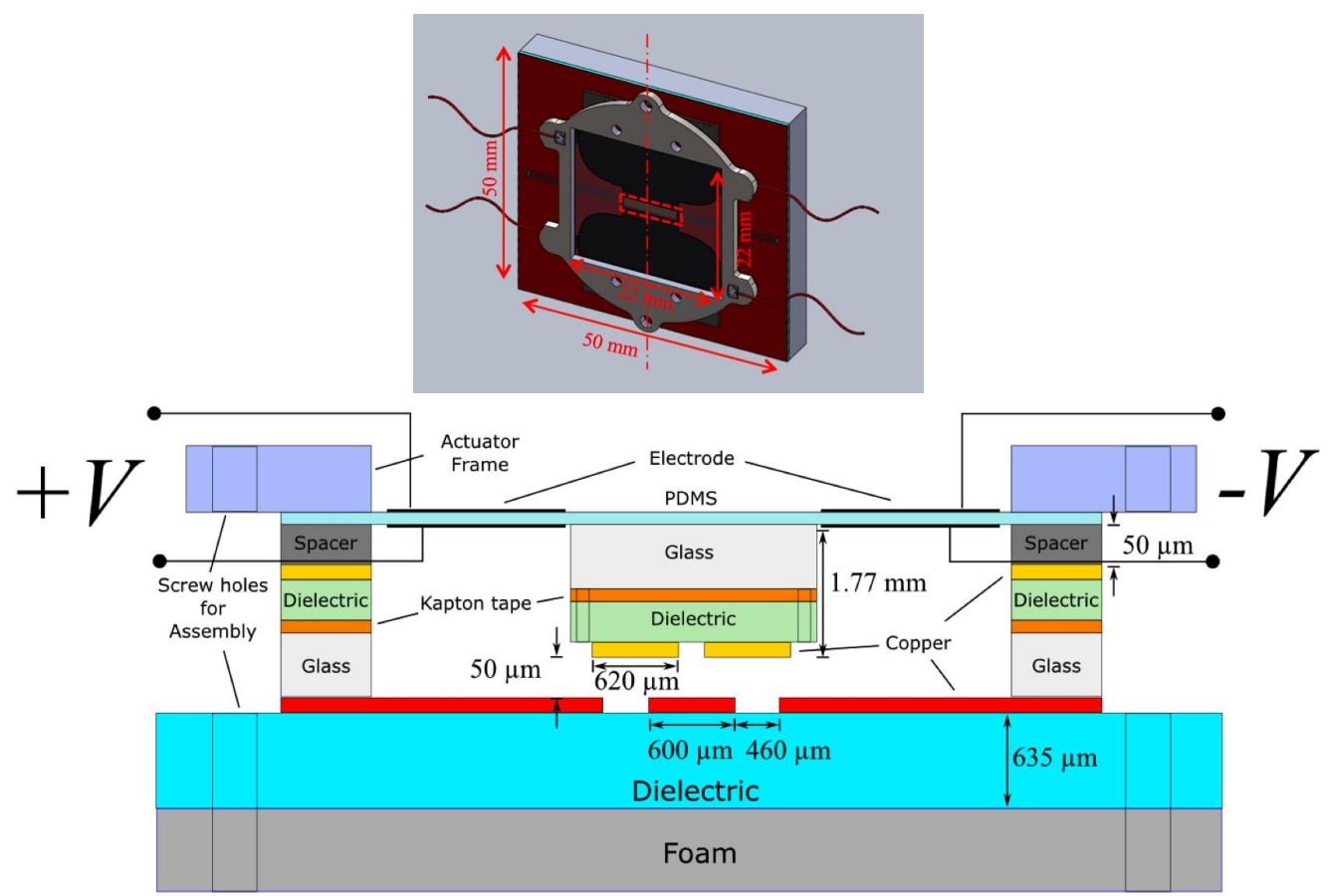

Figure 2. DEA phase shifter design. Top: Isometric view of CAD design (red dashed rectangle indicates EM active region). Bottom: Cross-section of device relative to centerline drawn in top figure.

requires accurate positioning and alignment to function optimally. Namely the air gap distance $z_{\text {gap }}$ is required to be 50 $\mu \mathrm{m} \pm 5 \mu \mathrm{m}$ for our current design. Moreover, EM simulations of the system predict substantial performance degradation if the loading lines and the CPW central transmission line are not parallel to each other to within $\sim 3^{\circ}$. The EM design also requires a minimum total lateral displacement of $500 \mu \mathrm{m}$ to produce the desired phase shift.

Our phase shifter device design can be seen in Figure 2. We use antagonist planar dielectric elastomer actuators (DEAs) to produce lateral displacements in the metallic loading lines. Two DEA segments are fabricated onto a single prestretched elastomer (PMDS) membrane by selectively patterning with a compliant electrode. This approach offers a low profile device, minimizing bulkiness. The activation of one of the electrodes increases the in-plane stretch in the activated segment and also relaxes the in-plane prestretch everywhere else in the membrane. Aside from increasing the mechanical energy in the elastomer, using prestretch increases the breakdown strength of the elastomer, however this also makes the membrane stiffer requiring a larger electric field to reach a given strain. The metallic loading lines, fixed to a passive region in the center of the membrane, are displaced linearly in-plane as a result of the actuation. The overall device size (approximately $50 \mathrm{~mm}$ by $50 \mathrm{~mm}$ excluding the alignment jig) represents a scale of device that could be conveniently fabricated using our current laboratory setup.

To minimize EM losses it is important that the EM active area (highlighted in Figure 2) stay free from materials with high EM absorption (such as carbon). For this reason a passive region is left in the center of the membrane. The use of two antagonist actuator segments rather than one larger segment also maximizes actuation strain whilst also preventing loss of tension occurring in the membrane. The electrode is tapered towards the edges in an attempt to minimize the effects of the fixed boundaries whilst maintaining as large an electrode area as possible. The prestretched elastomer membrane is bonded to a frame to retain its prestretch.

The metallic loading lines and CPW were fabricated by chemical etching of a commercially available dielectric laminated with copper (Rogers TMM10i, Rogers Corporation) with the desired pattern. The length of the loading was 10 $\mathrm{mm}$ for this initial prototype. The dielectric used was adhered to a glass substrate to facilitate bonding to the elastomer membrane by $\mathrm{O}_{2}$ plasma activation, and to maintain a level surface. A dense foam was placed underneath the CPW to 

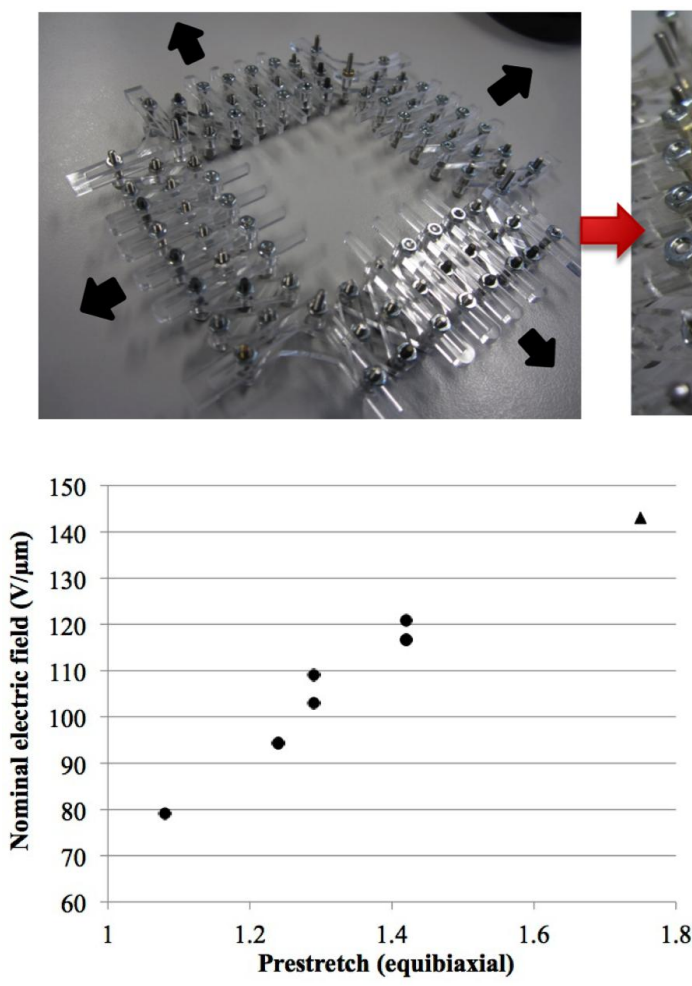

(b)
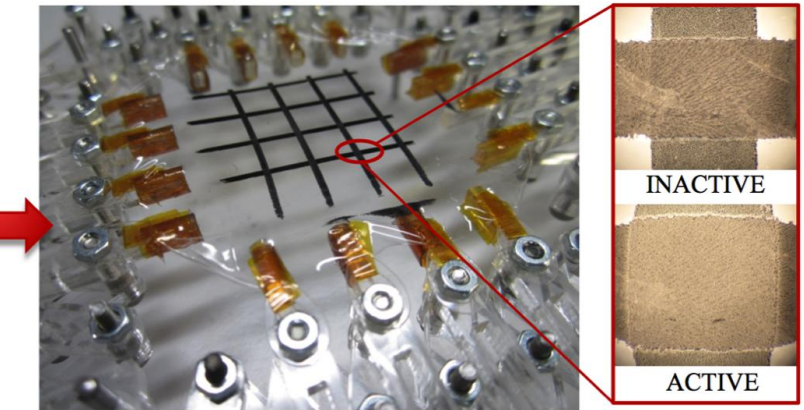

(a)

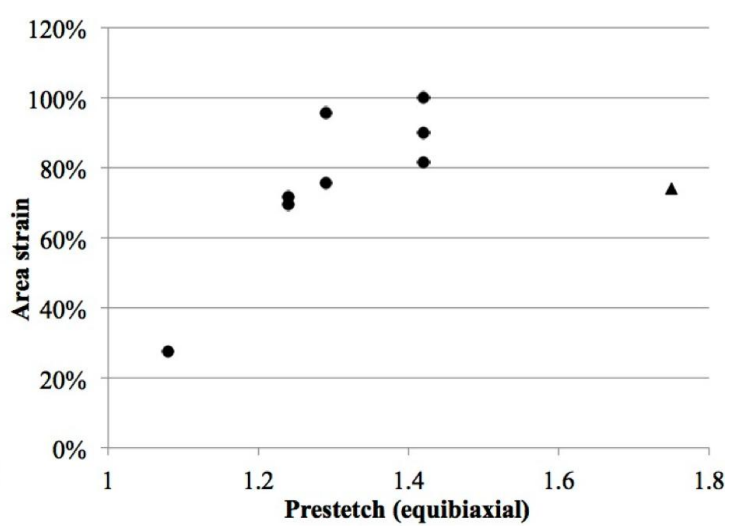

(c)

Figure 3. Characterization of nominal breakdown field, and area strain prior to breakdown, against equibiaxial prestretch for Sylgard 186 PDMS elastomer (Dow Corning). (a) Equibiaxial test rig and electrode pattern used in membrane characterization. (b) Results of the nominal breakdown field dependence against equibiaxial prestretch for several experiments. (c) Maximum actuation area strain prior to breakdown vs. prestretch. The nominal breakdown field increases monotonically with prestretch whereas the area strain in actuation appears to reach a maximum at 1.4 .

protect it during assembly. The loading elements were batched fabricated on the same substrate and then diced to dimensions $12 \mathrm{~mm}$ by $2 \mathrm{~mm}$. In order to ensure the correct spacing between the membrane and the transmission line plane, the membrane is placed on a unpatterned dielectric laminate bonded to a glass substrate stacked on top of a spacer made from laser cut $0.05 \mathrm{~mm}$ stainless steel shim (RS components), thus achieving the required $\mathrm{z}_{\mathrm{gap}}$ spacing (Figure 2). The PMMA is fixed to the CPW using screws which go through the actuator substrate, the CPW and the rigid foam.

Holes are drilled through the Kapton tape and dielectric of the loading element and are used to validate parallel alignment. The diameter of the holes is equal to the size of the gap between the ground plane and the central transmission line (TL). The actuator is moved manually relative to the CPW and the surface of the TL and viewed through holes using a microscope. Good alignment is attained when only the surface of the dielectric can be seen i.e. the copper of the TL and ground plane can no longer be seen (see Figure 8). These holes also enabled validation of the $z_{\text {gap }}$ spacing using digital holographic microscopy (see section 3 ).

\section{DEVICE FABRICATION AND ASSEMBLY}

\subsection{Device Fabrication}

To fabricate the dielectric elastomer membrane we use a two-component polydimethylsiloxane (PDMS) silicone elastomer (Sylgard 186, Dow Corning). The two PDMS components are mixed at a 10:1 ratio, with the addition of a 


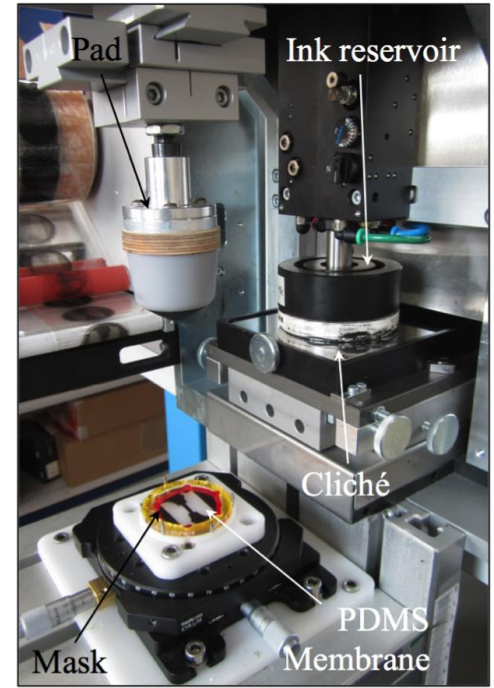

(a)

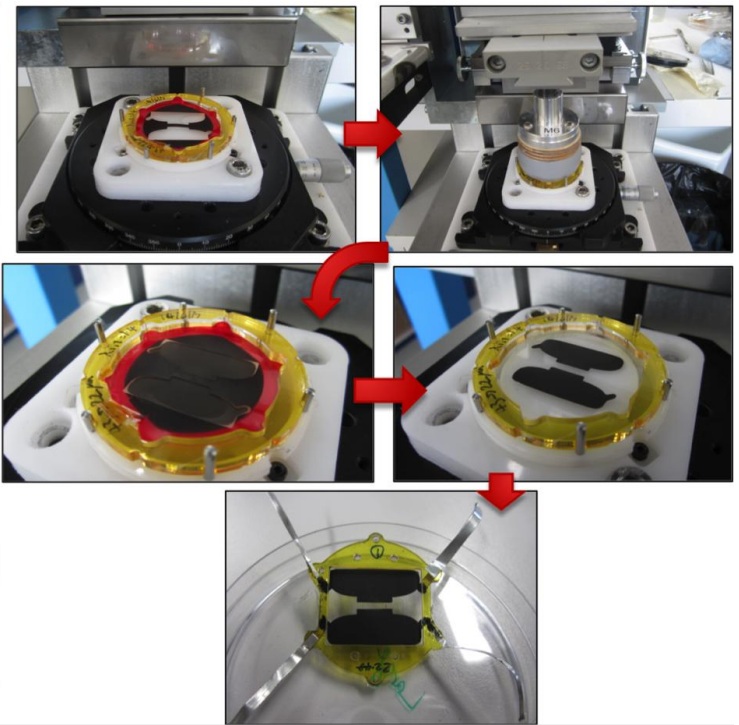

(b)

Figure 4. (a) Pad printing machine (Teca Print TPM101) used in electrode fabrication. (b) Select images from the pad printing process.

silicone solvent (OS-20, Dow corning - 4:1 PMDS:solvent ratio) to thin the mixture, using a planetary mixer (Thinky ARE-310). The mixer used also degasses the PDMS-solvent solution. The PDMS mixture is blade cast onto a PET substrate to a thickness of approximately $35 \mu \mathrm{m}$ using a Zehntner automatic film applicator coater. The PDMS is then allowed to cure in the oven for at least one hour at approximately $80^{\circ}$.

The cured elastomer membrane is cut into $40 \mathrm{~mm} \times 40 \mathrm{~mm}$ sections and prestretched equibiaxially. Many studies have reported on the effects of prestretch and electrode geometry on the electromechanical response of DEA membranes ${ }^{8,9,10}$. However, these studies consider axisymmetric DEAs (in area expansion mode) ${ }^{9}$, DEAs with a small active (electrode) region compared to the total membrane size ${ }^{8}$ or DEAs utilizing hanging masses ${ }^{10}$. The same analyses' are yet to be carried out for non-axisymmetric devices, constrained by rigid elements (i.e. the loading elements), optimized for unidirectional displacement, as is the case with our device. In an attempt to guide our design, a characterization of elastomer material was conducted to establish the nominal breakdown field and maximal strain response (area expansion) of our dielectric elastomer, as a function of prestretch. Though not indicative of the exact uniaxial strain that would be expected in our final device, it does give insight into the prestretch which optimally balances the increase in breakdown strength with membrane stiffening.

The characterization was performed using a purpose-built equibiaxial stretcher (Figure 3). A PDMS membrane was fixed to the stretcher and electrode lines approximately $1.5 \mathrm{~mm}$ wide were patterned on the membrane's top and bottom surfaces, and orientated orthogonal to each other, forming a grid of small DEAs (Figure 3(a)). A voltage bias was incrementally applied at each grid point in a row until breakdown. A different prestretch was applied to each row and the strain at each voltage increment was recorded using a microscope and digital camera. The initial thickness and prestretch thickness of the membrane was measured using a white light interferometry set-up. Figure 3(b) and Figure 3(c) show the results of the test. The last data point on both graphs was taken from ${ }^{8}$ where the same elastomer utilized here was used. The results show that although the breakdown field appears to increase with biaxial prestretch, the area strain produced appears to reach a maximum around a stretch of 1.4. Hence we chose to use a prestretch of 1.4 for our devices.

After prestretching, the membranes were then affixed to a rigid plastic holder lined with double-sided Kapton tape to maintain its prestretch. We fabricate our device electrodes via a stamping method using a pad-printing machine (Teca Print TPM101, Figure 4(a)). The machine operates by doctor blading small amounts of electrode "ink" onto a shallow reservoir etched into a metal plate called a cliché, which is subsequently picked up by a rubber pad (Figure 4(a)). In a standard procedure the cliché is etched with the desired electrode pattern, however we use a modified method where the 

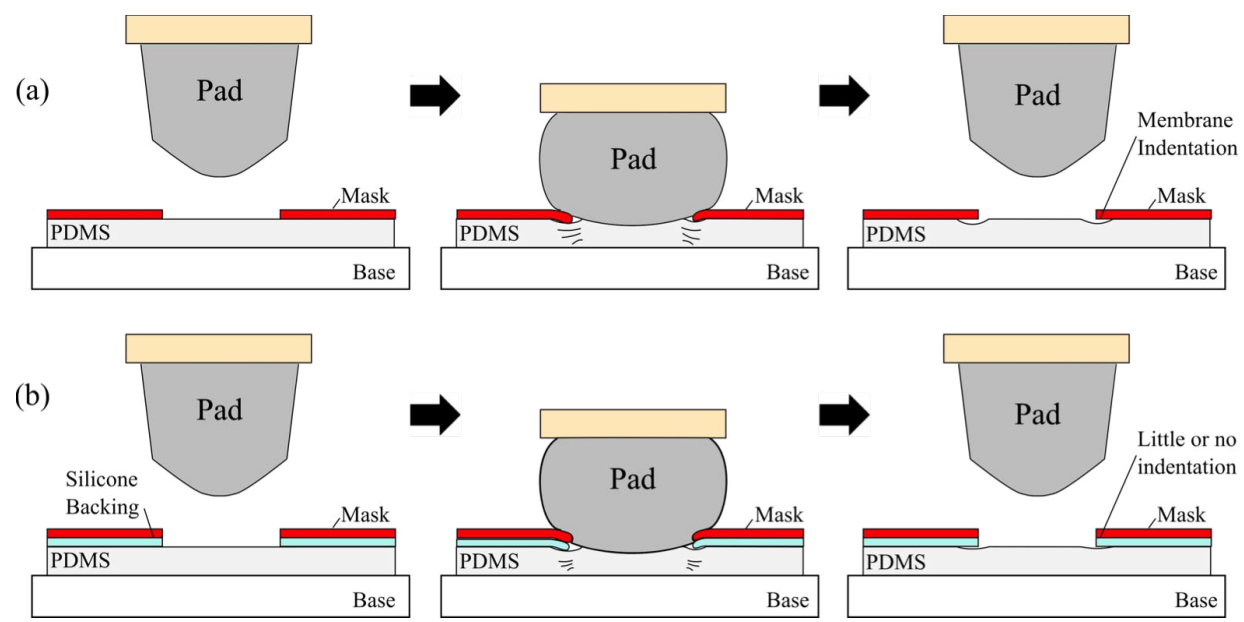

Figure 5. Schematic of pad printing process using a PET mask. (a) Without silicone backing underside. Potential cause of premature device failures identified as indentations produced in the membrane (right drawing) as the result of the mask pressing against the membrane (central drawing). (b) With silicone backing underside. Indentation on surface reduced or eliminated altogether.

cliché pattern is a circle (i.e. featureless) and the ink deposited through a laser cut PET mask placed on the membrane surface (Figure 4), thereby achieving the desired electrode geometry. This method facilitates fast prototyping and evaluation of designs. The shape of the perimeter of the mask is cut to match the internal shape of the rigid plastic frame used to retain the membranes prestretch. This pad-printing methodology ensured an electrode with high resolution (feature sizes down to $50 \mu \mathrm{m}$ attainable), ensuring the electrode material did not spill over into the central passive zone of the membrane.

Our first generation devices suffered from premature breakdown near the borders of the electrode. We believed this to be the result of indentations being produced in the membrane from the mask pressing down on it during the stamping process (Figure 5(a)). To alleviate this issue the mask was coated on its underside with a thin layer of PDMS prior to being laser cut. This significantly improved fabrication yield (Figure 5b)). We use ink composed of carbon black in a PDMS (Silbione LSR 4305, Bluestar Silicones) matrix.

The membrane is subsequently transferred to a smaller plastic frame before being placed with the loading elements into a plasma chamber (Zepto, Diener electronic) and exposed to oxygen plasma for approximately 20 seconds at $50 \%$ power. Radicals in the plasma first clean and then bind with the two surfaces making them highly reactive. The two activated surfaces are then removed from the chamber, placed in a jig to ensure alignment (Figure 6) and pressed in contact with each other for 1 minute. The device is left in the gig for a further 60 minutes to ensure a good bond quality.

The DEA membranes are mounted onto the CPW with the spacing elements and held in place using screws. The $\mathrm{z}_{\text {gap }}$ spacing was subsequently validated using a digital holographic microscope (DHM) (Lyncée tec), typically used for 3D real-time topography imaging at nanometric resolution. The DHM was used focused on the CPW surface and then on the top surface of the glass substrate of the loading element using it's working distance tool. This tool obtains vertical position at which the coherence is best for the two planes using a micron precision $\mathrm{x}-\mathrm{y}-\mathrm{z}$ stage. The difference between the vertical positions of the two surfaces subtracted by the thickness of the loading element (including the glass substrate and Kapton tape) determined the spacing $\mathrm{z}_{\text {gap }}$ (Figure 7). 


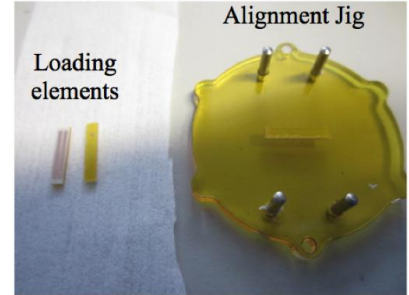

(a)

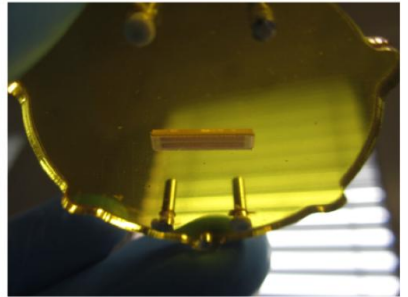

(b)

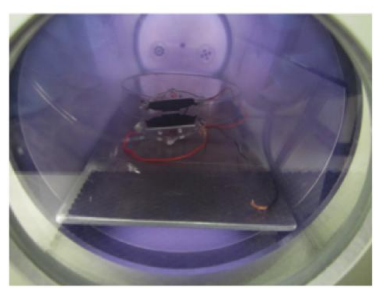

(c)

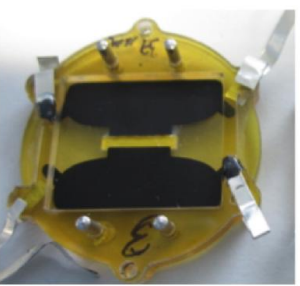

(d)

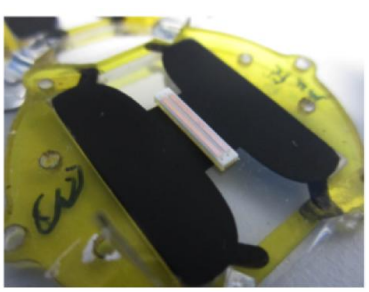

(e)

Figure 6. Bonding procedure of loading element to actuator membrane by $\mathrm{O}_{2}$ plasma activation. (a) Picture of loading element and alignment jig with shallow recess for placing loading element. (b) Loading element placed in alignment jig. (c) Actuator and loading element activated in $\mathrm{O}_{2}$ plasma chamber. (d) Loading element and actuator pressed in contact with each other and aligned using jig. (e) Bonded device.

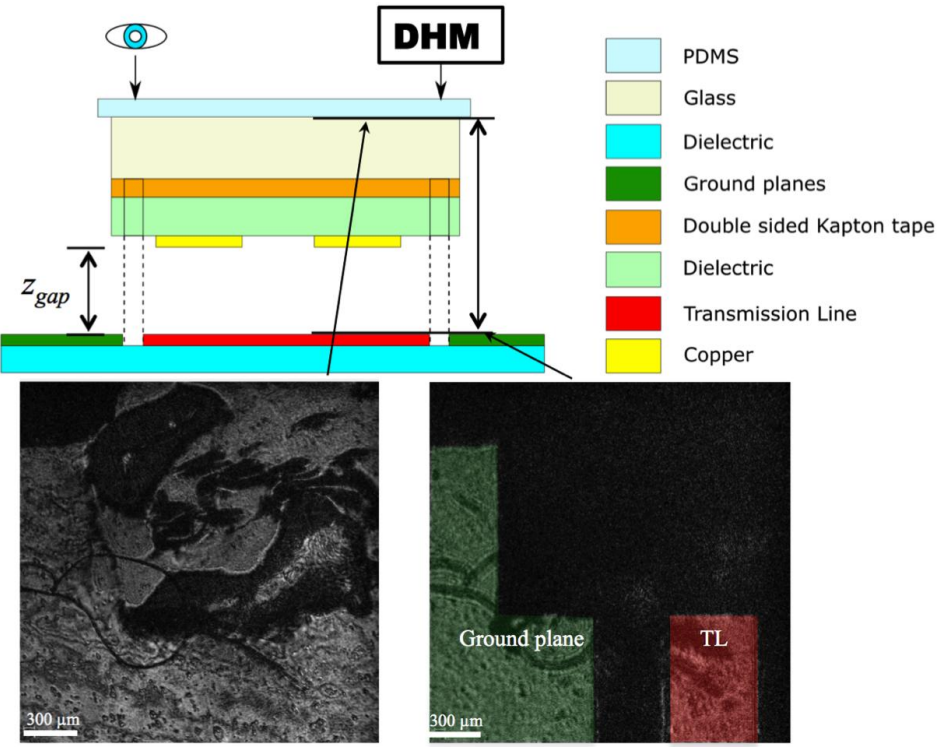

Figure 7. Validation of $\mathrm{z}_{\mathrm{gap}}$ spacing using a digital holographic microscope (DHM) (Lyncée tec). Top: Schematic of focusing planes. Bottom: Intensity images of the surfaces as seen through the DHM (green and red shading added to indicate the ground plane and transmission line respectively). The machine's working distance tool obtains vertical position at which the coherence is best for the two planes using a micron precision stage. The difference in the vertical position is used to determine the $\mathrm{z}_{\text {gap }}$ spacing by subtracting the thickness of the loading element, glass substrate and Kapton tape. 

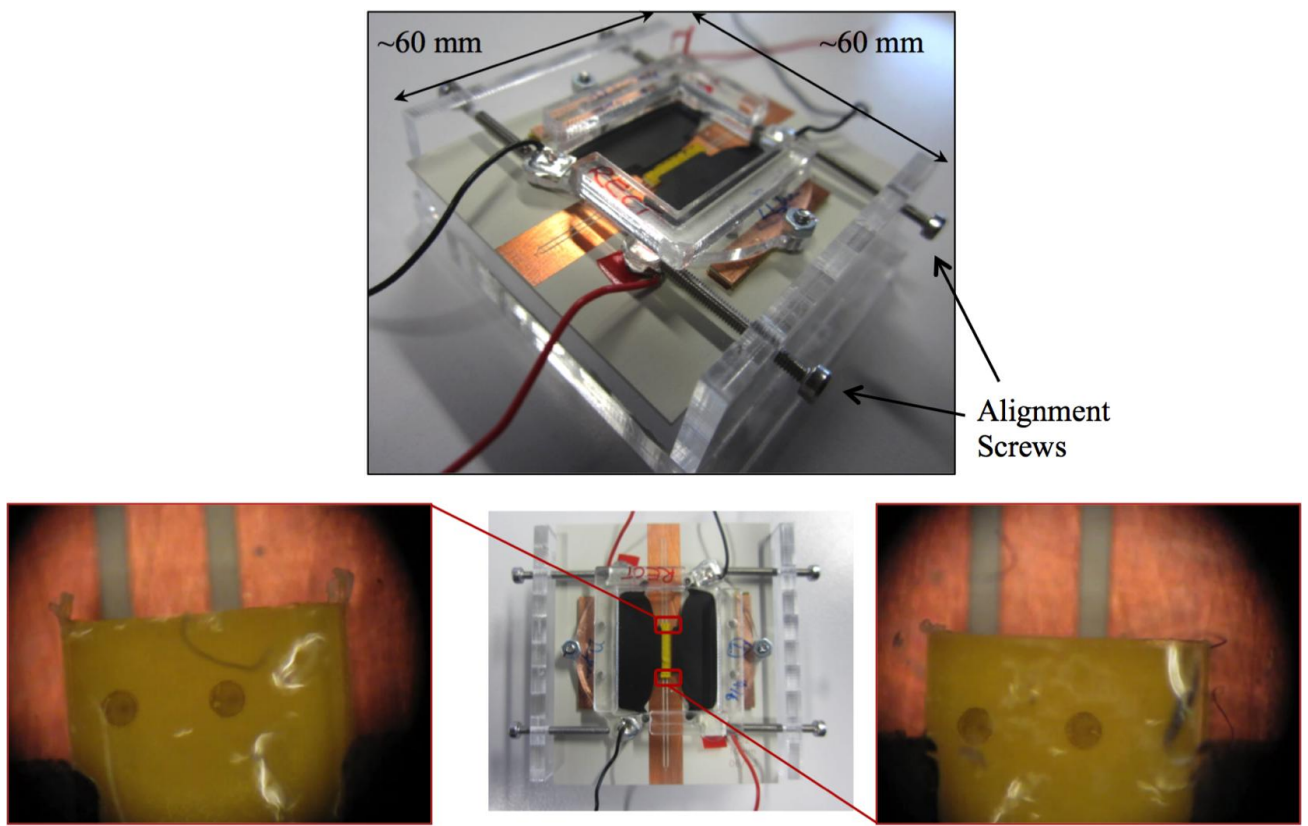

Figure 8. Top: Assembled device with alignment jig. Bottom: Example of alignment achieved using alignment methodology.

Once assembled the device was placed in an alignment jig which used four screws to adjust position of the actuator PMMA frame (Figure 8). The parallel alignment was validated using microscope with a digital camera (Figure 8), the screws were then used to lock the actuator frame in position once good alignment between the CPW and the loading element was attained.

\section{EXPERIMENTAL CHARACTERIZATION}

The test system used to make the MMW characterization unfortunately did not allow simultaneous measurement of EM properties and device displacement. Therefore the device displacement as a function of voltage was characterized before the MMW experiments using a microscope and digital camera (Figure 9(a)), and subsequently compared with EM simulations performed in Ansys HFSS. The phase at zero input voltage was measured for the device to correct for any misalignments produced during the assembly procedure, and the true position of the loading element relative to the CPW at the Central position (as shown in Figure 1(b)) inferred using the simulation results. Figure 9(b) shows the results of the MMW characterization at $25 \mathrm{GHz}$. The simulated result is shown with a solid blue line and the experimentally measured phase shift (for a given input voltage) shown with red asterisks. The voltage values indicated correspond to the input voltages used during the experiment, while $\Delta x$ ordinate value comes from pre-characterized displacement determined before the MMW experiment, as discussed above. Negative voltage values denote actuation of the right actuator segment and positive values as actuation of the right segment, as shown in Figure 2.

From Figure 9(a) it can be seen that the actuator was able to achieve the required total displacement of $500 \mu \mathrm{m}$ well within the safe operating range (max. electric field applied $\sim 60 \mathrm{~V} / \mu \mathrm{m}$, approximately $50 \%$ of the maximum by Figure 3 (b)). More importantly, Figure 9(b) shows that the device produced a large phase shift $\left(\sim 95^{\circ}\right)$ over the applied voltage range, being the first device of its kind to do so. Though the results for only the $25 \mathrm{GHz}$ frequency are shown here, this phase shifting was reproducible at other frequencies $(25-40 \mathrm{GHz})^{7}$. The experimental values agree very well with the simulation for the negative voltage inputs (actuation of the left actuator segment) but agree less well with the positive voltage inputs (actuation of the right actuator segment). We assume this to be the result of a discrepancy between the pre-measured displacement of the actuator and the displacement produced during the experiment, most likely arising 


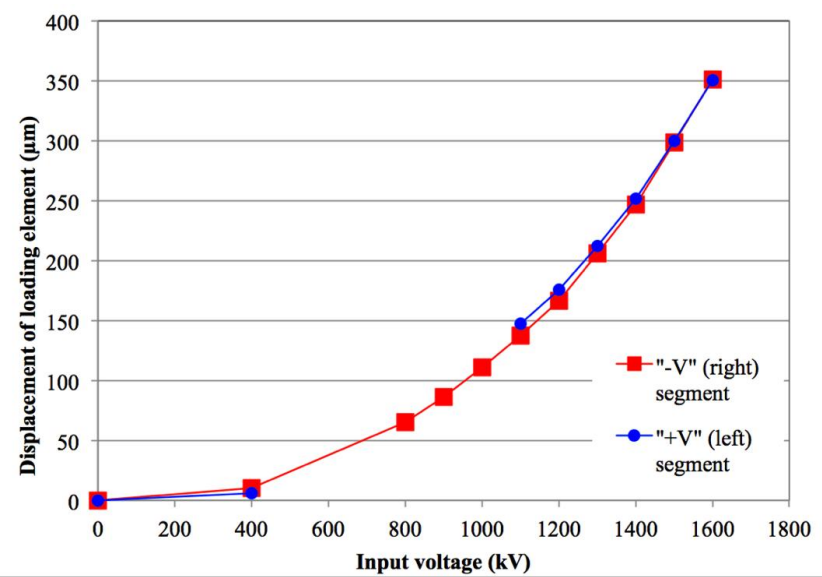

(a)

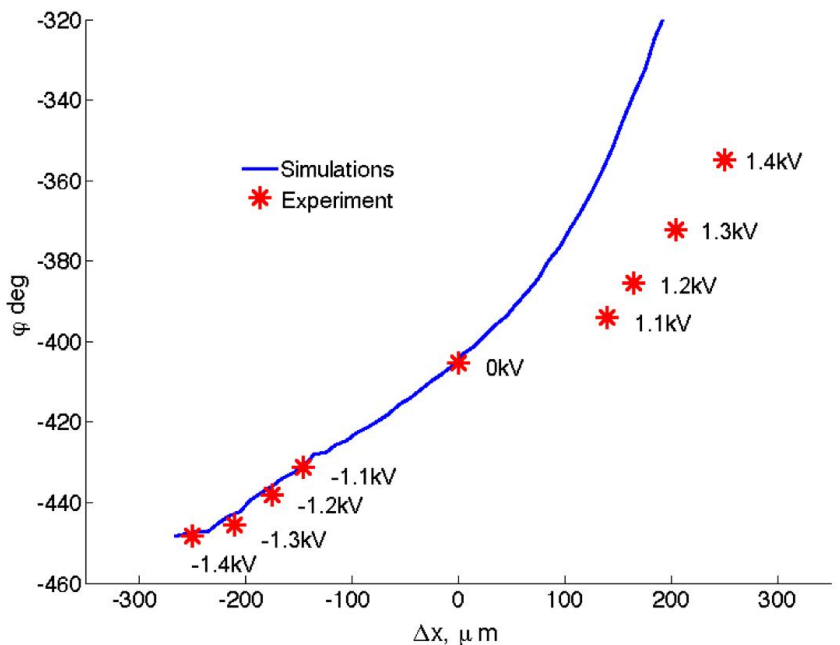

(b)

Figure 9. (a) Results of pre-characterization of loading element displacement as a function of the applied voltage performed prior to device MMW characterization (portion of $+V$ curve missing due to an error with the data). (b) Results of MMW characterization at $25 \mathrm{GHz}$. Blue solid line is the EM simulation and the red asterisks are the experimental phase shift for a given input voltage. The $\Delta x$ ordinate is determined by a pre-characterization of the device displacement (a)

during transportation and test preparation. In future device designs self-sensing ${ }^{11}$ or closed-loop control ${ }^{12}$ could be incorporated for accurate determination or specification of actuator displacement.

The phase-shift per EM loss, the most important figure of merit for true-time-delay phase shifters, was measured to be $\sim 235^{\circ} / \mathrm{dB}$ (at $35 \mathrm{GHz}$ ). This represents state of the art performance, considerably out-performing other phase shifter technologies, such as MMIC phase shifters, at similar frequencies.

\section{CONCLUSION}

We have developed the first MMW phase shifter driven by DEAs. The device is made using robust yet inexpensive materials and fabrication processes. Our device successfully achieves analogue phase shifting within the applied voltage range, and demonstrates state of the art phase shift per loss of $\sim 235^{\circ} / \mathrm{dB}(35 \mathrm{GHz})$. The success of our device paves the way for other MMW reconfiguration concepts using DEAs. 


\section{ACKNOWLEDGEMENTS}

This research was supported by the European Space Agency (NPI project REACT) and the Swiss National Science Foundation (SNSF) under Grant No. 133583 and 200020_140394.

\section{REFERENCES}

[1] Arrebola, M., Encinar, J.A., Cahill, R., and Toso, G., "Dual-reflector antenna with a reflectarray subreflector for wide beam scanning range at 120GHz," in 2012 Int. Conf. Electromagn. Adv. Appl., 848-851 (2012).

[2] Volakis, J.L., [Antenna Engineering Handbook], 4th ed., McGraw-Hill Professional (2007).

[3] Hum, S.V., and Perruisseau-Carrier, J., "Reconfigurable Reflectarrays and Array Lenses for Dynamic Antenna Beam Control: A Review," IEEE Transactions on Antennas and Propagation 62(1), 183-198 (2014).

[4] Kim, M., Yang, J.G., and Yang, K., "Switched transmission-line type Q-band 4-bit MMIC phase shifter using InGaAs pin diodes," Electronics Letters 46(3), 219 (2010).

[5] Yang, J.G., and Yang, K., "Ka-Band 5-Bit MMIC Phase Shifter Using InGaAs PIN Switching Diodes," IEEE Microwave and Wireless Components Letters 21(3), 151-153 (2011).

[6] Pelrine, R., Kornbluh, R., Pei, Q., and J., J., "High-Speed Electrically Actuated Elastomers with Strain Greater Than 100\%," Science 287(5454), 836-839 (2000).

[7] Romano, P., Araromi, O., Rosset, S., Shea, H., and Perruisseau-Carrier, J., "Tunable millimeter-wave phase shifter based on dielectric elastomer actuation,” Applied Physics Letters 104(2), 024104 (2014).

[8] Akbari, S., Rosset, S., and Shea, H.R., "Improved electromechanical behavior in castable dielectric elastomer actuators," Applied Physics Letters 102(7), 071906 (2013).

[9] Koh, S.J.A., Li, T., Zhou, J., Zhao, X., Hong, W., Zhu, J., and Suo, Z., "Mechanisms of large actuation strain in dielectric elastomers," Journal of Polymer Science Part B: Polymer Physics 49(7), 504-515 (2011).

[10] Lu, T., Huang, J., Jordi, C., Kovacs, G., Huang, R., Clarke, D.R., and Suo, Z., "Dielectric elastomer actuators under equal-biaxial forces, uniaxial forces, and uniaxial constraint of stiff fibers," Soft Matter 8(22), 6167 (2012).

[11] Gisby, T.A., O’Brien, B.M., and Anderson, I.A., "Self sensing feedback for dielectric elastomer actuators," Applied Physics Letters 102(19), 193703 (2013).

[12] Rosset, S., O’Brien, B.M., Gisby, T., Xu, D., Shea, H.R., and Anderson, I.A., "Self-sensing dielectric elastomer actuators in closed-loop operation," Smart Materials and Structures 22(10), 104018 (2013). 